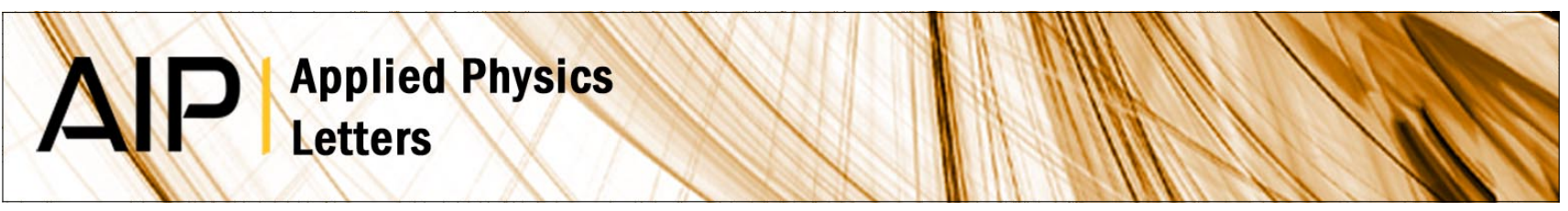

\title{
Resonant cavity modes in gallium oxide microwires
}

Iñaki López, Emilio Nogales, Bianchi Méndez, and Javier Piqueras

Citation: Appl. Phys. Lett. 100, 261910 (2012); doi: 10.1063/1.4732153

View online: http://dx.doi.org/10.1063/1.4732153

View Table of Contents: http://apl.aip.org/resource/1/APPLAB/v100/i26

Published by the American Institute of Physics.

\section{Related Articles}

Thermoelectric properties of p-type (Bi2Te3)x(Sb2Te3)1-x single crystals doped with $3 w t . \%$ Te J. Appl. Phys. 113, 073709 (2013)

Dielectric function of $\mathrm{Cu}(\mathrm{In}, \mathrm{Ga}) \mathrm{Se} 2$-based polycrystalline materials

J. Appl. Phys. 113, 063505 (2013)

High-pressure synthesis and in-situ high pressure $\mathrm{x}$-ray diffraction study of cadmium tetraphosphide J. Appl. Phys. 113, 053507 (2013)

Dielectric and transport properties of bismuth sulfide prepared by solid state reaction method J. Appl. Phys. 113, 043704 (2013)

Decoupling free-carriers contributions from oxygen-vacancy and cation-substitution in extrinsic conducting oxides J. Appl. Phys. 113, 033706 (2013)

\section{Additional information on Appl. Phys. Lett.}

Journal Homepage: http://apl.aip.org/

Journal Information: http://apl.aip.org/about/about_the_journal

Top downloads: http://apl.aip.org/features/most_downloaded

Information for Authors: http://apl.aip.org/authors

\section{ADVERTISEMENT} \section{AIP $\begin{gathered}\text { Applied Physics } \\ \text { Letters }\end{gathered}$}

\section{EXPLORE WHAT'S NEW IN APL}

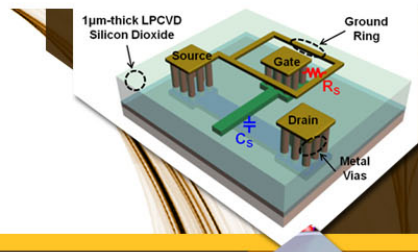

SURFACES AND INTERFACES

Focusing on physical, chemical, biological structural, optical, magnetic and electrical properties of surfaces and interfaces, and more.. 


\title{
Resonant cavity modes in gallium oxide microwires
}

\author{
Iñaki López, Emilio Nogales, Bianchi Méndez, and Javier Piqueras \\ Departamento de Física de Materiales, Universidad Complutense de Madrid, Madrid 28040, Spain
}

(Received 2 February 2012; accepted 14 June 2012; published online 28 June 2012)

\begin{abstract}
Fabry Perot resonant modes in the optical range $660-770 \mathrm{~nm}$ have been detected from single and coupled $\mathrm{Cr}$ doped gallium oxide microwires at room temperature. The luminescence is due to chromium ions and dominated by the broad band involving the ${ }^{4} \mathrm{~T}_{2}-{ }^{4} \mathrm{~A}_{2}$ transition, strongly coupled to phonons, which could be of interest in tunable lasers. The confinement of the emitted photons leads to resonant modes detected at both ends of the wires. The separation wavelength between maxima follows the Fabry-Perot dependence on the wire length and the group refractive index for the $\mathrm{Ga}_{2} \mathrm{O}_{3}$ microwires. @ 2012 American Institute of Physics. [http://dx.doi.org/10.1063/1.4732153]
\end{abstract}

In the last years, the properties derived from light confinement into semiconducting micro and nanostructures and their potential applications have attracted considerable interest. In particular, luminescence and waveguiding properties of single nanowires have been reported in view of their applications as lasers or efficient optical cavities in photonic devices. ${ }^{1-12}$ The high crystalline quality and the commonly flat surfaces of nanowires are valuable points to consider them very good candidates to explore their behavior as optical resonators. Fabry-Perot (FP) modulations due to reflections between wire flat ends and whispering gallery modes (WGMs) due to total internal reflections in microstructures with regular polyhedral cross-sections were reported in some semiconducting micro and nanostructures. FP resonant modes from near band edge transitions have been reported in $\mathrm{GaN}^{1,2}$ GaAs, ${ }^{3}$ and $\mathrm{ZnO}$ (Refs. 4 and 5) nanowires. FP and WGM resonant modes in defect-related emission bands have been observed in $\mathrm{ZnO}$ and $\mathrm{In}_{2} \mathrm{O}_{3}$ microwires with rectangular, hexagonal, ${ }^{7-9}$ or polyhedral ${ }^{10}$ cross-sections. It is worth exploring the coupling phenomena in more complex structures, such as branched or crossing wires, in order to provide applications of light confinement in semiconductor nanostructures. However, there are still few works devoted to this issue, e.g., Refs. 11 and 12. On the other hand, the choice of the material is an important factor, which determines both the spectral range of emission, the nature of the luminescence, and even their feasibility to operate at room temperature.

$\beta-\mathrm{Ga}_{2} \mathrm{O}_{3}$ micro and nanowires have been revealed as attractive host for optically active impurities. The wide band gap $(4.9 \mathrm{eV})$ enables to tune their luminescence properties from the ultraviolet to infrared regions by controlling the defect structure and/or impurity doping. On the other hand, $\mathrm{Ga}_{2} \mathrm{O}_{3}$ has a rather high refractive index suitable for light confinement and waveguiding behavior, which could lead to optical gain in a resonant cavity. In particular, we have studied in previous works the waveguiding behavior of rare earth (RE) and $\mathrm{Cr}$ doped $\mathrm{Ga}_{2} \mathrm{O}_{3}$ nanowires. ${ }^{13-15}$ In this work, we demonstrate the quality of $\mathrm{Ga}_{2} \mathrm{O}_{3}: \mathrm{Cr}$, Sn codoped microwires as cavity resonators. Upon laser excitation, light generated from optically active $\mathrm{Cr}$ impurities is confined into the microwire leading to characteristic resonances in the wavelength range between $640-770 \mathrm{~nm}$. The resonant modes were explored in isolated straight and bended wires, branched wires, and crossing wires.
The Sn doped microwires were grown on a gallium oxide pellet, by thermal oxidation at $1100^{\circ} \mathrm{C}$ of metallic gallium in the presence of tin oxide under argon gas flow in a tubular furnace at atmospheric pressure. A further thermal treatment at $1500^{\circ} \mathrm{C}$ for $15 \mathrm{~h}$ is driven to incorporate $\mathrm{Cr}$ ions by diffusion into the structures. After the growth, the structures were removed from the pellet and placed onto a silicon wafer without the need of any glue. The purpose of Sn doping is to favour the growth of long and often branched wires, suitable to investigate the waveguiding and optical resonator properties. The mechanism of Sn influence on the final morphology of the wires will not be discussed here but we take advantage of the morphology of the codoped wires to study the cavity effects not only in single wires but also in crossing structures.

Morphological characterization has been carried out in a FEI Inspec S50 scanning electron microscope (SEM). Micro-photoluminescence ( $\mu$-PL) spectra and imaging have been performed in a Horiba Jobin Ybon LabRam HR800 Raman confocal microscope. The excitation light was the $325 \mathrm{~nm}$ line of a He-Cd laser. A custom design module has been used to deviate the excitation laser spot from the collection point. ${ }^{11}$ All PL measurements were performed at room temperature.

XRD measurements (not shown) confirm that the crystal structure of the synthesized wires is the monoclinic $\beta-\mathrm{Ga}_{2} \mathrm{O}_{3}$ (JCPDS 00-041-1103). Figure 1(a) shows a SEM image of Sn doped $\mathrm{Ga}_{2} \mathrm{O}_{3}$ wires on gallium oxide pellet. After the $\mathrm{Cr}$ diffusion process, the structures retain their morphology. Figure 1(b) shows two interconnected wires of 133 and $97 \mu \mathrm{m}$ length with polyhedral cross-sections of 2.2 and $1.35 \mu \mathrm{m}$ width, respectively. Their cross-sections are uniform along the axis and the tip has a triangular shape, as shown in the inset of Figure 1(b). Energy dispersive x-ray microanalysis in the SEM detects the Cr peak in the codoped structures (see supplementary information) ${ }^{21}$ but quantification of the dopant concentration is difficult to achieve as it is close to the system detection limit (in the range of 0.5 at. \%).

Figure 2(a) shows two crossing wires, labeled W1 and $\mathrm{W} 2$. The widths of $\mathrm{W} 1$ and $\mathrm{W} 2$ are around $1.85 \mu \mathrm{m}$ and $1 \mu \mathrm{m}$, respectively. Their ending points have been numbered from 1 to 4. Under UV excitation, red luminescence is generated by the $\mathrm{Cr}$ ions in the gallium oxide matrix and it corresponds to the sharp characteristic R lines at $696 \mathrm{~nm}$ and to 

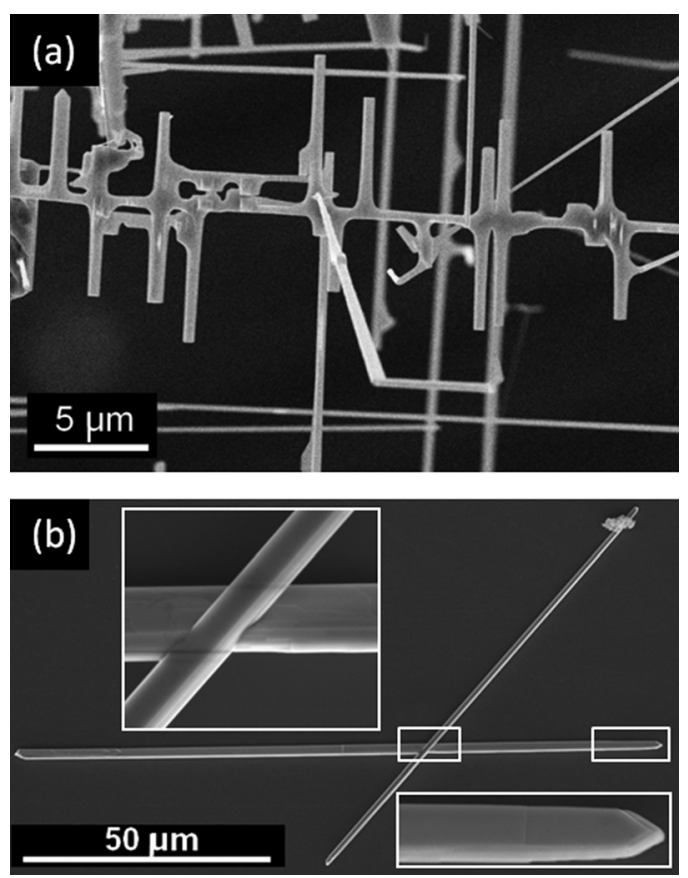

FIG. 1. SEM micrographs of (a) Sn doped $\mathrm{Ga}_{2} \mathrm{O}_{3}$ structures and (b) two $\mathrm{Cr}, \mathrm{Sn}$ codoped interconnecting wires. Insets: details of the crossing point and of the wire extreme.

the ${ }^{4} \mathrm{~T}_{2}-{ }^{4} \mathrm{~A}_{2}$ phonon assisted broad band observed at room temperature. ${ }^{16}$ The waveguiding behavior of excited light is clearly observed. When UV excitation is applied to the upper end of W1 (point 2 in Fig. 2(a)), red PL is split and reaches both ends (points 1 and 4 ) of W1 and W2 as shown by the arrows in Figure 2(b). In addition, a bright red spot is observed at point 3 (upper end of W2). This observation can be explained by the coupling of the evanescent field of the red PL at the contact region, ${ }^{12,17}$ so that the $\mathrm{Cr}$ related, red PL generated in W1 is transferred to W2 and confined in both wires. The light output at upper end of W2 (point 3) may come from the reflection at the opposite end. In addition, a fraction of the UV incident light transmitted along W1 would be coupled with W2 at the contact area and excite red PL in W2. This wire behaves as a waveguide and, consequently, red spots are observed in both extremes of W2.
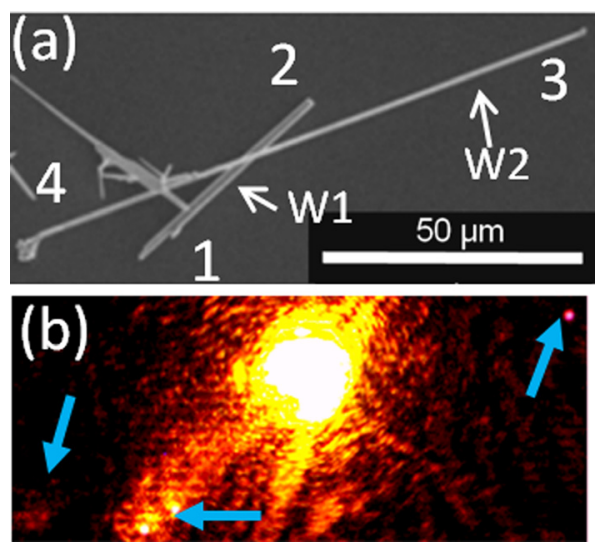

FIG. 2. (a) SEM image of two crossing nanowires with their corresponding labels. (b) Micro-PL image under excitation at point 2. Arrows highlight the ending points.
In order to study the behavior of $\mathrm{Ga}_{2} \mathrm{O}_{3}$ microwires as optical resonators, we have analyzed the PL spectra of the red light at the ending faces of the interconnected wires shown in the Figure 1(b), which show triangular shape ends. Figure 3 shows their corresponding micro-PL image. By UV excitation at the crossing point, red PL is generated and guided in the two rods and red spots are seen at the four ends, as expected according to the above discussion. MicroPL spectra from these wires show very interesting features. With the special setup in our confocal microscope, we have recorded spectra at wire ends during excitation at the crossing point. Figure 3(b) shows the characteristic $\mathrm{Cr}^{3+}$ emission acquired at $\mathrm{A}$ end. A careful inspection of this spectrum reveals a clear modulation spreading over the whole emission band (inset Figure 3(b)). The separation between adjacent maxima, $\Delta \lambda$, is $0.98 \mathrm{~nm}$. The inset also shows the modulation observed at B point of $1.24 \mathrm{~nm}$ at $\lambda=725 \mathrm{~nm}$. PL spectra from the opposite extremes of each wire show the same modulation. We have also checked that the observed modulation does not depend on the excitation point and is characteristic of each wire. In principle, these wires could act as Fabry-Perot optical cavities of length $\mathrm{L}$ with modes $\mathrm{N}=2 n \mathrm{~L} / \lambda$, where $\mathrm{N}$ is an integer and $n$ is the refractive index, if we consider their ends smooth enough to operate as reflecting optical mirrors. ${ }^{18}$ We have estimated the cavity length from this condition with data from PL spectra in Figure 3(b). By choosing, for example, two consecutive modes $\left(\lambda_{\mathrm{N}}=725 \mathrm{~nm}\right.$ and $\left.\lambda_{\mathrm{N}+1}=726 \mathrm{~nm}\right)$ and the refractive index from literature of about $1.91,{ }^{19}$ the FP condition for the cavity length gives a value of $137 \mu \mathrm{m}$, in good concordance with the SEM images.
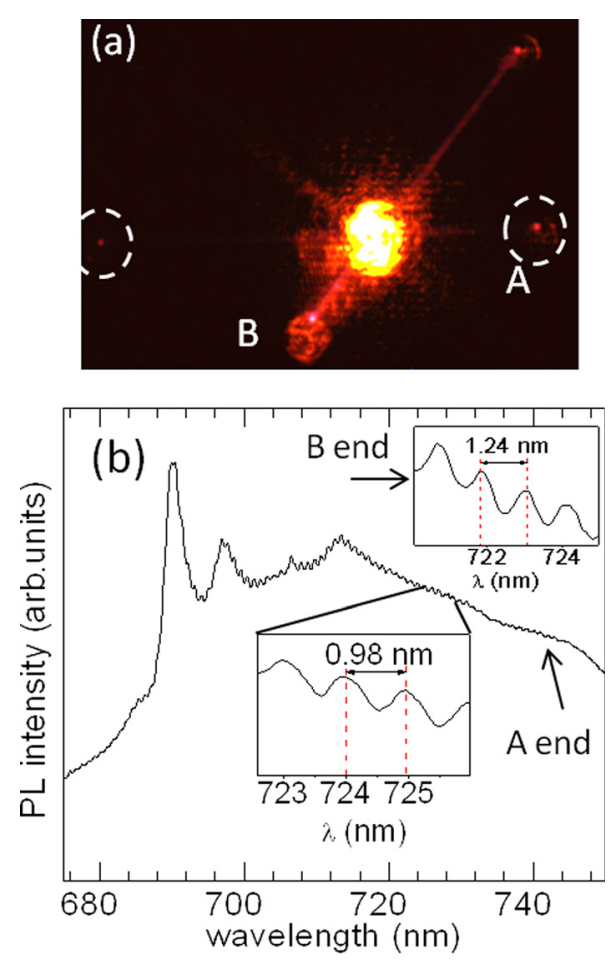

FIG. 3. (a) Micro-PL image of two $\mathrm{Sn}, \mathrm{Cr}$ codoped $\mathrm{Ga}_{2} \mathrm{O}_{3}$ crossing wires under laser excitation at crossing point. (b) PL spectra from A point showed in (a). Insets show the separation between adjacent maxima around $725 \mathrm{~nm}$ for each wire. 


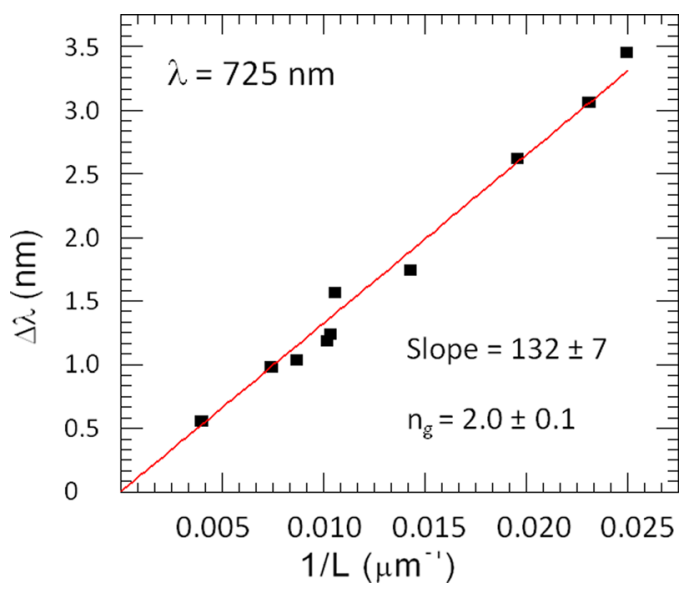

FIG. 4. Spacing between adjacent modes vs. $1 / L$ for $\lambda=725 \mathrm{~nm}$, which shows a linear dependence. The refractive index group is obtained from the slope of the linear fitting curve.

Similar measurements were made on several wires of different lengths in order to correlate the modulation with the wire length, which is a characteristic feature of optical cavities. Figure 4 shows the graph that represents $\Delta \lambda$ vs. $1 / \mathrm{L}$, for ten wires, where a clear linear dependence is observed. For Fabry-Perot cavity modes, the mode spacing $\Delta \lambda$ for a cavity with length $\mathrm{L}$ is given by $\Delta \lambda=\lambda^{2} /\left(2 \mathrm{~L} n_{g}\right)$, where $n_{g}$ is the group refractive index that is defined as $n-\lambda \mathrm{d} n / \mathrm{d} \lambda .^{18}$ By fitting data of Figure 4 to a linear function, the experimental value $1.99 \pm 0.10$ of the group refractive index of gallium oxide is obtained. This value is in agreement with previously experimental data and Cauchy dispersion model fit. ${ }^{19}$ Therefore, the data support that the observed modulation is related to Fabry-Perot resonances in the wires. Additionally, quality factor $\mathrm{Q}$ and finesse $\mathrm{F}$ factor were estimated for several microwires. $Q$ is a measure of stored photon energy within the cavity and $\mathrm{F}$ is directly dependent on the resonator losses, which could provide information about the reflectivity of end faces, as is the main loss mechanism in the wires. They are calculated from $\mathrm{Q}=\lambda / \Gamma$ and $\mathrm{F}=\Delta \lambda / \Gamma$, where $\Gamma$ is the fullwidth at half maximum of the peak at $\lambda$. From $\Gamma$ values of several maxima in the PL spectra of several wires, $Q$ values of about 1500 and 1330, respectively, are obtained. Then, $\mathrm{Ga}_{2} \mathrm{O}_{3}$ wires are suitable host for storing photon energy. However, finesse $\mathrm{F}$ values obtained are about $2.7 \pm 0.2$ in both wires, which indicates that losses at end faces of these wires are large.

The occurrence of FP resonances would be of interest as $\mathrm{Cr}$ doped $\mathrm{Ga}_{2} \mathrm{O}_{3}$ wires could be used for lasing applications. The broad emission band provides a rather wide range for resonance wavelengths in microstructures, which has been considered for tunable lasers. $^{20}$ This feature is derived from phonon assisted process in the luminescence mechanisms, usually present in materials with high Huang-Rhys factor. It should be also mentioned that the concentration of $\mathrm{Cr}$ ions into gallium oxide wires is very low, below the detection limit of standard microanalysis systems. However, a strong red emission, visible with naked eye in the present experiments, is achieved from individual wires at room temperature. In addition, their morphology with uniform thickness and flat surfaces prevents optical losses and enhances optical confinement of guided modes. It has been reported than in nanowire lasers, mirror losses overpass waveguide losses due to their small reflection coefficient. ${ }^{20} \mathrm{~A}$ recent work reported that the threshold gain to achieve laser action in $\mathrm{ZnO}$ nanowires is strongly dependent on length and diameter, showing that very thin nanowires never reach the threshold, independent of the nanowire length. ${ }^{5}$ Hence, further work with the aim of improving the balance between optical gain and losses in $\mathrm{Ga}_{2} \mathrm{O}_{3}$ wires will be made in the next future.

In summary, single codoped $\mathrm{Ga}_{2} \mathrm{O}_{3}: \mathrm{Sn}, \mathrm{Cr}$ straight structures with waveguiding behavior and light confinement have been studied with the aid of a confocal microscope. The characteristic red PL of $\mathrm{Cr}^{3+}$ in $\beta-\mathrm{Ga}_{2} \mathrm{O}_{3}$, observed at room temperature, is confined in gallium oxide microwires. Guided modes follow the path of the wire or couple to other contacting wires. The presence of resonant modes has been observed by recording PL spectra at the end points of several wires. The separation between modes scales with the inverse of the length wire according to the Fabry-Perot law for resonant optical cavities, and the group refractive index of gallium oxide has been determined. Estimation of Q factor was made for several wires, leading to rather high values.

This work has been supported by MICINN through Projects MAT 2009-07882 and Consolider Ingenio CSD 200900013 and by BSCH-UCM (Project GR35-10A-910146).

${ }^{1}$ J. C. Johnson, H. J. Choi, K. P. Knutsen, R. D. Schaler, P. D. Yang, and R. J. Saykally, Nature Mater. 1, 106 (2002).

${ }^{2}$ S. Gradecak, F. Qian, Y. Li, H.-G. Park, and C. M. Lieber, Appl. Phys. Lett. 87, 173111 (2005).

${ }^{3}$ B. Hua, J. Motohisa, Y. Ding, S. Hara, and T. Fukui, Appl. Phys. Lett. 91, 131112 (2007).

${ }^{4}$ J. C. Johnson, H. Yan, P. Yang, and R. J. Sakally, J. Phys. Chem. B 107, 8816 (2003).

${ }^{5}$ M. A. Zimmler, J. Bao, F. Capasso, S. Muller, and C. Ronning, Appl. Phys. Lett. 93, 051101 (2008).

${ }^{6}$ X. Ye, H. Mao, J. Wang, and Z. Zhu, Appl. Phys. Lett. 99, 261112 (2011).

${ }^{7}$ C. Czekalla, C. Sturm, R. Schmidt-Grund, B. Cao, M. Lorenz, and M. Grundmann, Appl. Phys. Lett. 92, 241102 (2008).

${ }^{8}$ J. Li, S. Lee, Y. H. Ahn, J.-Y. Park, K. H. Koh, and K. H. Park, Appl. Phys. Lett. 92, 263102 (2008).

${ }^{9}$ H. Dong, Z. Chen, L. Sun, J. Lu, W. Xie, H. Tan, C. Jagadish, and X. Shen, Appl. Phys. Lett. 94, 173115 (2009).

${ }^{10}$ H. Dong, S. Sun, L. Sun, W. Xie, L. Zhou, X. Shen, and Z. Chen, Appl. Phys. Lett. 98, 011913 (2011).

${ }^{11}$ R.-M. Ma, X.-L. Wei, L. Dai, S.-F. Liu, T. Chen. S. Yue, Z. Li, Q. Chen, and G. G. Qin, Nano Lett. 9, 2697 (2009).

${ }^{12}$ Y. Xiao, C. Meng, X. Wu, and L. Tong, Appl. Phys. Lett. 99, 023109 (2011).

${ }^{13}$ E. Nogales, B. Méndez, and J. Piqueras, Nanotechnology 19, 035713 (2008).

${ }^{14}$ E. Nogales, J. A. García, B. Méndez, and J. Piqueras, Appl. Phys. Lett. 91, 133108 (2007).

${ }^{15}$ E. Nogales, B. Méndez, J. Piqueras, and J. A. García, Nanotechnology 21, 115201 (2009).

${ }^{16}$ E. Nogales, J. A. García, B. Méndez, and J. Piqueras, J. Appl. Phys. 101, 033517 (2007).

${ }^{17}$ T. Voss, G. T. Svacha, S. Müller, C. Ronning, D. Konjhodzic, F. Marlow, and E. Mazur, Nano Lett. 7, 3675 (2007).

${ }^{18}$ X. Duan, Y. Huang, R. Agarwal, and C. M. Lieber, Nature (London) 421, 241 (2003).

${ }^{19}$ M. Rebien, W. Henrion, M. Hong, J. P. Mannaerts, and M. Fleischer, Appl. Phys. Lett. 81, 250 (2002).

${ }^{20}$ A. V. Maslov and C. Z. Ning, Appl. Phys. Lett. 83, 1237 (2003).

${ }^{21}$ See supplementary material at http://dx.doi.org/10.1063/1.4732153 for $\mathrm{x}$-ray microanalysis measurements. 